\title{
Primary Synovial Sarcoma of the Mediastinum: A Case Report and Review of the Literature
}

Ouji $\mathbf{M}^{1}$, Souissi $\mathbf{S}^{2}$, Mbarek $\mathbf{K}^{3}$, Koubaa $\mathbf{M}^{4}$ and Mezghani Boussetta $\mathbf{S}^{1 *}$

${ }^{1}$ Department of Radiology, Regional Hospital of Ben Arous, Tunisia

${ }^{2}$ Department of Pulmonology, Regional Hospital of Ben Arous, Tunisia

${ }^{3}$ Department of Emergency, Regional Hospital of Ben Arous, Tunisia

${ }^{4}$ Department of Pathology, Habib Thameur Hospital, Tunis, Tunisia

\section{Abstract}

Background: Synovial sarcomas most commonly occur in the soft tissues of the extremities of young adults. A primary occurrence in the mediastinum is very rare with only a few reported cases in the world literature.

Study aims: to insist on the rarity of this pathology, its imaging features and the importance for recognizing unusual presentation of this aggressive neoplasm to aid appropriate clinical management.

Cases: Here in, we report a rare case of primary monophasic spindle cell synovial sarcoma of the mediastinum in a 31-year-old man who had an incidental finding of such a tumor. The imaging studies on admission showed $12 \mathrm{~cm}$ anterior- middle mediastinal mass with multiple metastases in the lung, pleura, mediastinal lymph nodes and spine. An ultrasound-guided percutaneous needle biopsy of a cervical extension of the mass permitted histopathology and immuno histochemical analysis which supported the diagnosis of monophasic spindle cell synovial sarcoma. The patient was treated by chemo-therapy without response.

Conclusion: A wide range of neoplasms both primary and metastatic occurs in the mediastinum which poses considerable diagnostic difficulties. A synovial sarcoma should be considered in the differential diagnosis. Imaging can guide percutaneous needle biopsy of the tumor and evaluate its stage. Immunohistochemistry can be very helpful for an accurate diagnosis.

Keywords: Mediastinum; Synovial sarcoma; CT; Immunohistochemistry

\section{Introduction}

Synovial sarcoma derives its name from its histomorphological resemblance to synovium. However this is a misnomer because more recent evidence suggests that the tumor derives from primitive pluripotential mesenchyme which is capable of synovial differentiation. Histologically, classical synovial sarcoma combines a mixture of epitheloid and spindle cell components exhibiting a biphasic pattern. If this tumor shows only one cell type it can be classified as monophasic spindle cell type or monophasic epithelial cell type [1]. Synovial sarcomas are uncommon but well defined soft tissue tumors accounting for 5 to $10 \%$ of all soft tissue sarcomas. These tumors occur in adolescent and young adults of $15-40$ years of age and more than $85 \%$ of synovial sarcoma arises in the extremities, mainly in the lower limbs around the knee. Although this type of tumors has been reported in unusual areas like head and neck, lower back, pleura and peritoneum, synovial sarcoma of the mediastinum is very rare [2-4].

Here we report a case of a monophasic spindle cell primary synovial sarcoma of the mediastinum by insisting on its imaging and pathological features.

\section{Case Report}

A 31 year old man nonsmoker with no past medical history presented to the emergency room for acute chest pain after a minor trauma occurred on the same day. He had no respiratory symptoms. There was no history of cough, dyspnoea or weight loss. Physical examination was unremarkable. Chest $\mathrm{X}$ ray revealed a large mediastinal mass, with tracheal displacement and interruption of the aortic arch profile. It results in opacity of homogeneous appearance without excavation or apparent calcifications, its lateral edges were sharp and regular; it was at level of the anterior-middle compartment on the lateral view and was associated with multiple secondary lung nodes centimeter. There was no pleural effusion (Figure 1). Contrast Enhanced Computed Tomography (CECT) revealed a $12 \times 8 \mathrm{~cm}$, well defined mass within the anterior- middle compartment of the mediastinum which was homogenous without cystic or necrosis areas.

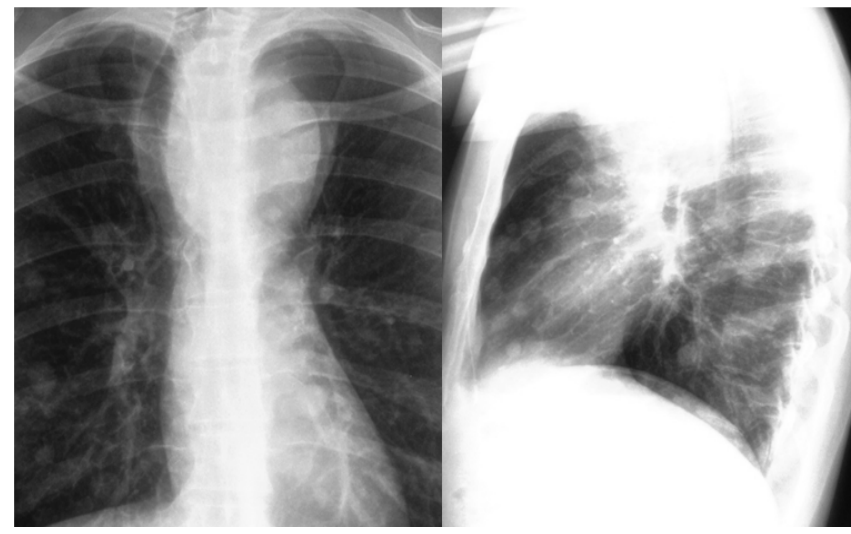

Figure 1: $\mathrm{PA}$ and lateral chest $\mathrm{x}$ ray show a mass of the anterior-middle mediastinum displacing the trachea to the right side with multiple metastatic pulmonary nodules.
${ }^{*}$ Corresponding author: Mezghani Boussetta S, The faculty of Medicine of Tunis, Department of Radiology, University EL manar 2, 15 Rue Djebel Alkhdhar, La Rabita 1007, Tunis, Tunisia, Tel: +216 71872 253; E-mail: sana.boussetta@rns.tn

Received May 27, 2015; Accepted June 23, 2015; Published June 24, 2015

Citation: Ouji M, Souissi S, Mbarek K, Koubaa M, Mezghani Boussetta S (2015) Primary Synovial Sarcoma of the Mediastinum: A Case Report and Review of the Literature. J Clin Case Rep 5: 548. doi:1 0.4172/2165-7920.1000548

Copyright: (c) 2015 Ouji M, et al. This is an open-access article distributed under the terms of the Creative Commons Attribution License, which permits unrestricted use, distribution, and reproduction in any medium, provided the original author and source are credited. 
The mass showed peripheral calcifications and low enhancement. It was closely related to the trachea and gross vascular structures compressing them. The mass extended through the cervico-thoracic junction to the neck causing compression of left lobe of the thyroid gland without invasion. Enlarged mediastinal lymphadenopathy of the sub-carena space was detected and multiple secondary nodes of lungs and pleura were seen. Moreover, computed tomography scan revealed nodular lytic lesions in the spine to suspect bone metastases (Figure 2). There was no hepatic or other abdominal metastases. The patient had no history or evidence on clinical or radiographic examination of tumor elsewhere, supporting of a primary origin at this site.

Ultrasound-guided percutaneous needle biopsy of the cervical extension of the tumor was performed which on gross consisted of four fragments fully tumor, the longest measuring $13 \mathrm{~mm}$. Histologically the tumor was composed of monomorphous spindle cells with dense cellularity showing an interlascing fascicular arrangement, hyaline and fibrilar areas and hemangio-pericytoma like vasculature. The cells showed an oval or rounded nucleus which was small in size and

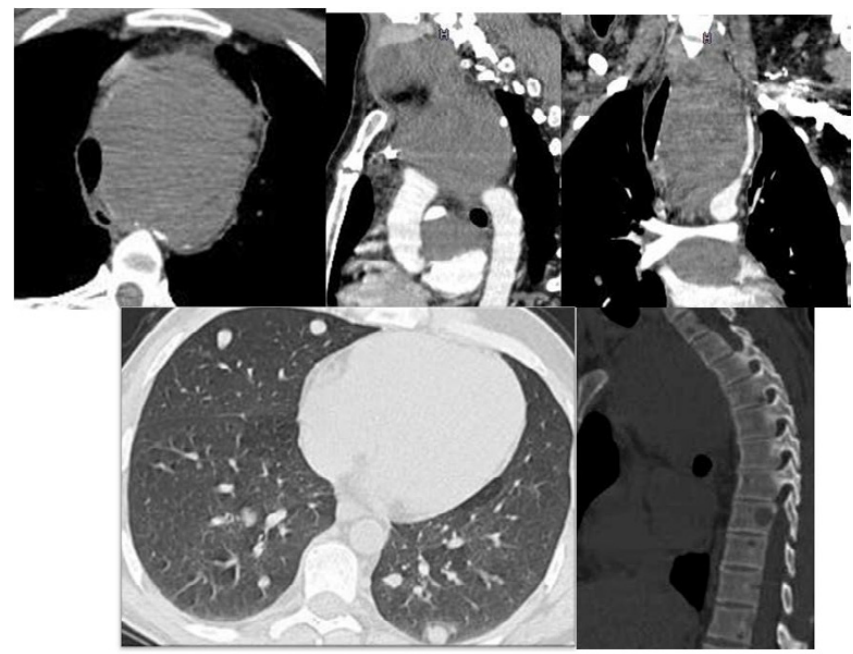

Figure 2: Computed tomography demonstrates a large well defined homogeneous anterior -middle mediastinal mass compressing and displacing vascular and aero-digestive structures. The mediastinal mass shows a cervical extension displacing the left thyroid lobe.

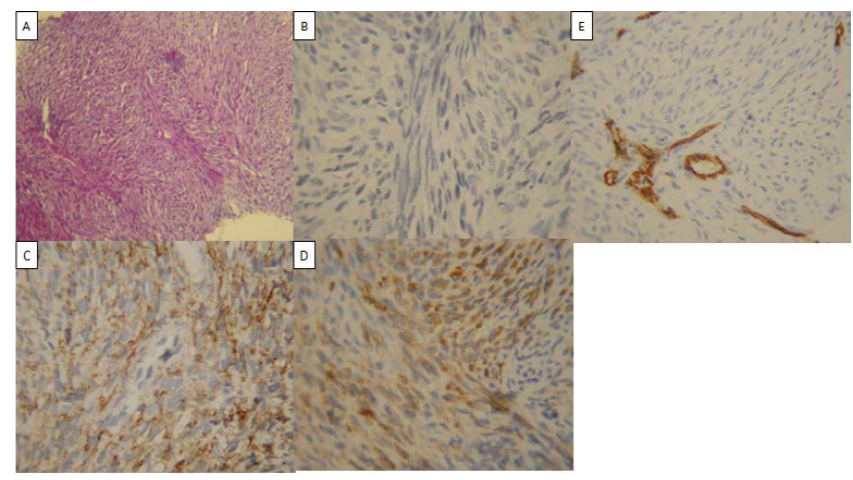

Figures 3: (A-D) Histologic and immuno-histologic features;

A) Photo micrograph showing a densely cellular spindle cell interlacing fascicles $(H$ and $E \times 100)$. B) The tumor cells were negative for CytoKeratin (AE1, AE3). C) a diffuse immuno-reactivity was seen with EMA. D) A diffuse positivity with variable intensity was seen for bcl-2; E) Tumor cells were negative for CD34. Note the hemangiopericytoma like of vasculature. chromatic. Mitosis index was about 10 per 10 high power fields. It has not been found area of tumoral necrosis. On immunohistochemistry, the tumor cells stained with anti-EMA and bsl2 and did not stain with anti-keratin AE1/AE3, CD 34 and S100 protein (Figure 3). In conclusion, the histological and immuno-histological diagnosis was that of a monophasic spindle cell synovial sarcoma at least intermediate grade.

The patient was treated by chemo-therapy with Doxorubicin and Ifosphamide without response. He died of tumor six months after diagnosis.

\section{Discussion}

Synovial sarcoma is a rare and aggressive malignant soft tissue tumour which most commonly occurs in the extremities of young adults. Intra thoracic involvement of synovial sarcoma including lung, pleura, chest wall locations is unusual $[5,6]$. Although synovial sarcoma has been reported to metastasis to the mediastinum, its occurrence as primary neoplasm in this location is rare and has only recently been recognized. Only a few reports and series of primary synovial sarcoma of the mediastinum are described in the literature and all reported cases does not exceed forty so far [2-4,6-15].

In 1989, Witkin et al reported, for the first time, 4 cases of biphasic tumors of the mediastinum with features of synovial sarcoma [2]. The patients were all adult men who presented with solitary mediastinal masses in the region of the aortic arch or in perihilar region. All tumors histologically displayed a biphasic growth pattern. Again later, Suster et al. [4], through a series of 15 cases of primary synovial sarcoma of the mediastinum, confirmed previous observations on these tumors and documented the existence of a monophasic spindle cell variant in the mediastinum. Other subtypes are uncommon including the undifferentiated type [6].

Intra thoracic synovial sarcomas are clinically often a diagnostic challenge due to their non specific presentation. Common symptoms include chest pain, shortness of breath, hemoptysis and cough. However, most patients present with a slow growing, painless mass, in a way similar to our case $[6,8,13]$.

Radiographic examination should include chest radiography, Computed Tomography and Magnetic Resonance Imaging (MRI) of the tumor. On chest radiographs, the lesion is typically uniform with well-circumscribed rounded or lobulated borders $[4,6,13]$.

Computed Tomography demonstrates a well defined homogenous or heterogeneously enhancing mass containing necrotic areas and soft tissue components. Peripheral or spotty calcifications were described in some cases. Invasion and infiltration of the surrounding tissue can be present on Computed Tomography scan in patients with advanced stages of such tumors. Ipsilateral pleural effusion is common. Lymphadenopathies are rare $[4,6,12,13]$.

Compared with soft tissue synovial sarcoma, primary pulmonary and mediastinal synovial sarcoma shows less vascularity and a similar triple sign (bright, dark and gray) representing tumor, hemorrhage, and necrosis on magnetic resonance imaging. The T2 weighted images on MRI demonstrate well the so called" triple sign". Synovial sarcoma typically grows in centrifugal fashion and can also compress surrounding structures $[4-6,13]$.

In this case, at the time of initial diagnosis, the tumor was large, located in anterior- middle mediastinum. It had homogeneous density with peripheral calcifications and showed less vascularity. Tumor had 
close relationship with gross vessels and was accompanied by invasion of pleura, lymphatic nodes and lungs. Distant metastases to the spine were very probably.

In the study of Suster including fifteen cases, the tumors were distributed throughout all mediastinal compartments. Six cases were located in the posterior mediastinum and three occupied the anteriormiddle mediastinum with compromise of hilar structures and great vessels, as was the case with our patient. Three of the cases located in the posterior mediastinum showed focal infiltration and destruction of ribs and vertebrae [4].

It had been reported some cases arising from the pericardium which posed difficulties for surgical resection $[14,15]$.

A case of primary mediastinal synovial sarcoma with transdiaphragmatic extension had been reported by Al-Rajhi and Korula $[16,17]$, in our case the tumor presented a cervical extension through the cervico-thoracic junction; in our knowledge, this is the only case reported with this feature. This one had allowed practicing ultrasound guided needle-biopsy, offering the clue of diagnosis. Generally, Computed Tomography has been the modality of choice for guidance of needle-biopsy of mediastinal tumors; this enables better preoperative planning and possibly "en bloc resection" of the mass.

Accurate staging of the disease is important for appropriate patient management and requires the evaluation of the primary tumor and assessment for distant disease. Enhanced Computed Tomography and MRI has been the traditional imaging tool for assessment of this type of pathology but more recently, Positron Emission Tomography (PET CT) were used showing increased $18 \mathrm{~F}-\mathrm{FDG}$ uptake by the primary tumor, it permitted to identify other foci that may represent metastatic disease. Combined PET CT imaging is likely to become the imaging modality of choice for follow up of patients with these rare tumors $[4,8,11]$.

Immunohistochemistry is essential to differentiate between monophasic synovial sarcoma from other spindle cell tumors in the absence of molecular tests for diagnosis. The tumor cells were positive for CAM5.2, cytokeratin7, bcl 2 and vimentin. They were negative for S 100, CD 34, CD 99, desmin and SMA. With this findings, especially positive keratin and bcl 2, it is possible to exclude two entities that can most closely resemble monophasic synovial sarcoma in this location, solitary fibrous tumor (CD 34 positive) and malignant peripheral nerve sheath tumor (focally S100 positive) $[4,6,13]$. These two tumors were excluded in this case as the tumour cells were negative for CD34 and S 100 respectively.

In equivocal cases, ultrastructural examination coupled with demonstration of the characteristic $(\mathrm{X} ; 18)$ chromosomal translocation involves in synovial sarcoma may be the only means for establishing a definitive diagnosis $[1,2,4,6]$. Cyto-genetic techniques were not used in this case.

A wide variety of clinical and histological features have been reported to be of prognostic significance. Young age ( $<15$ years), tumor size $(<5 \mathrm{~cm})$, distal extremity location, and low tumor stage have been shown to have more favorable outcome.

All of the reported sarcomas of the mediastinum were large and tended to recur However survival up to 14 years has been documented. The prognosis of synovial sarcomas of the mediastinum is even poorer than that of synovial sarcoma of the extremities $[18,19]$.

Broad surgical resection is the corner stone of therapy. Complete resection of the tumor with good margins was the overwhelming factor in determining survival in a review of primary mediastinal sarcomas. Conservative surgery and adjuvant radiotherapy is favored when complete surgical excision is not possible. Radiotherapy is often required to obtain local control of the disease with adjuvant chemotherapy $[4,13,19]$.

The place for chemotherapy of this tumor is not well defined. Combination of adriamycin and ifosfamid has been used as an adjuvant therapy and for recurrences in patient with atypical location and size superior or equal to $5 \mathrm{~cm}$. The role of palliative chemotherapy for unresectable or advanced disease is not clear, but this is common practice [19]. Unlike this case, a recent publication has reported a very favorable outcome with neoadjuvant chemo-therapy based on Doxorubicin and Ifosfamid in a total of 6 cycles, tumor reduction was subtotal, it was possible to exceed an acute course of the disease and a completely resection of the tumor was possible under good operating conditions thereafter [20].

\section{Conclusion}

Synovial sarcoma must be considered in the differential diagnosis of spindle cell tumors arising in the mediastinum. This case report emphasis the importance of an accurate staging of the disease using Computed Tomography, the utility of percutaneous imaging guided needle biopsy and the importance of an accurate histopathological diagnosis and use of appropriate immunohistochemical markers in the diagnosis of this unusual tumour in an unusual site. It confirmed the poor prognostic of this tumor which arose immediately at an advanced stage.

\section{References}

1. Fisher C (1986) Synovial sarcoma: ultrastructural and immunohistochemica features of epithelial differentiation in monophasic and biphasic tumors. Hum Pathol 17: 996-1008

2. Witkin GB, Miettinen M, Rosai J (1989) A biphasic tumor of the mediastinum with features of synovial sarcoma. A report of four cases. Am J Surg Pathol 13: 490-499.

3. Trupiano JK, Rice TW, Herzog K, Barr FG, Shipley J, et al. (2002) Mediastinal synovial sarcoma: report of two cases with molecular genetic analysis. Ann Thorac Surg 73: 628-630.

4. Suster S, Moran CA (2005) Primary synovial sarcomas of the mediastinum: a clinicopathologic, immunohistochemical, and ultrastructural study of 15 cases. Am J Surg Pathol 29: 569-578.

5. Duran-Mendicuti A, Costello P, Vargas SO (2003) Primary synovial sarcoma of the chest: radiographic and clinicopathologic correlation. J Thorac Imaging 18: $87-93$

6. Hartel PH, Fanburg-Smith JC, Frazier AA, Galvin JR, Lichy JH, et al. (2007) Primary pulmonary and mediastinal synovial sarcoma: a clinicopathologic study of 60 cases and comparison with five prior series. Mod Pathol 20: 760-769.

7. Hsieh PP, Ho WL, Peng HC, Lee T (2002) Synovial sarcoma of the mediastinum Zhonghua Yi Xue Za Zhi (Taipei) 65: 83-85.

8. Cheng LS, Tse GM, Li WW, Lee TW, Yim AP (2003) Mediastinal synovial sarcoma: a case report and literature review. Can Respir J 10: 393-395.

9. Gotoh M, Furukawa S, Motoishi M, Fujimoto T, Okazaki T, et al. (2004) Synovial sarcoma of the mediastinum: report of a case. Surg Today 34: 521-524.

10. Migeon-Duballet I, Albert-Dunais V, Milinkevitch S, Paccalin M (2005) [Mediastinal synovial sarcoma in the elderly: a case report]. Rev Med Interne 26: 435-436.

11. Jeganathan R, Davis R, Wilson L, McGuigan J, Sidhu P (2007) Primary mediastinal synovial sarcoma. Ulster Med J 76: 109-111.

12. Kaira K, Ishizuka T, Sunaga N, Hashimoto K, Yanagitani N, et al. (2008) Primary mediastinal synovial sarcoma: a report of 2 cases. J Comput Assist Tomogr 32: 238-241. 
Citation: Ouji M, Souissi S, Mbarek K, Koubaa M, Mezghani Boussetta S (2015) Primary Synovial Sarcoma of the Mediastinum: A Case Report and Review of the Literature. J Clin Case Rep 5: 548. doi:1 0.4172/2165-7920.1000548

13. Henninger B, Freund M, Zelger B, Putzer D, Bonatti H, et al. (2009) Primary mediastinal synovial sarcoma: a case report and review of the literature. Cases J 2: 6948.

14. Pal M, Ghosh BN, Roy C, Manna AK (2010) Posterior mediastinal biphasic synovial sarcoma in a 12 year-old boy: a case report and review of literature. $J$ Cancer Res Ther 6: 564-566.

15. Ravikumar G, Mullick S, Ananthamurthy A, Correa M (2011) Primary synovial sarcoma of the mediastinum : a case report. Case Rep Surg 2011: 602853.

16. Al-Rajhi N, Husain S, Coupland R, McNamee C, Jha N (1999) Primary pericardial synovial sarcoma: a case report and literature review. J Surg Oncol 70: 194-198.
17. Korula A, Shah A, Philip MA, Kuruvila K, Pradhip J, et al. (2009) Primary mediastinal synovial sarcoma with transdiaphragmatic extension presenting as a pericardial effusion. Singapore Med J 50: e26-28.

18. Ferrari A, Gronchi A, Casanova M, Meazza C, Gandola L, et al. (2004) Synovia sarcoma: a retrospective analysis of 271 patients of all ages treated at a single institution. Cancer 101: 627-634.

19. Rosen G, Forscher C, Lowenbraun S, Eilber F, Eckardt J, et al. (1994) Synovial sarcoma. Uniform response of metastases to high dose ifosfamide. Cancer 73: 2506-2511.

20. Balieiro MA, Lopes AJ, Costa BP, Veras GP, Perelson PS, et al. (2013) The surprising outcome of a giant primary mediastinal synovial sarcoma treated with neoadjuvant chemotherapy. J Thorac Dis 5: 94-96. 